\title{
Atomic Resolution Study of Defects in Graphene
}

\author{
Jamie H. Warner
}

\section{Department of Materials, University of Oxford, Parks Road, Oxford, OX1 3PH, United Kingdom}

Defects in graphene influence its chemical, electrical, mechanical and magnetic properties. It is therefore important to have a deep understanding of their exact atomic structure and the most common forms. The introduction of defects into the bulk pristine lattice of graphene breaks up the uniform symmetry of the hexagonal lattice structure. Although the removal of atoms in graphene forms vacancies, atoms can reconfigure their position to maintain bonding to three nearest neighbour atoms, as opposed to the edge atoms. Bond rotations often play a major role in reducing strain and results in more complex low energy vacancy structures than one would initial expect.

High quality monolayer graphene is produced by chemical vapour deposition on a $\mathrm{Cu}$ catalyst and transferred onto specialized silicon nitride TEM grids for imaging. The atomic structure of graphene can be directly imaged using low-voltage aberration corrected transmission electron microscopy. Using Oxford's JEOL2200MCO aberration-corrected transmission electron microscopy at an accelerating voltage of $80 \mathrm{kV}$ has enabled several fundamental defect structures to be resolved, such as the monovacancy and tetravacancy [1,2]. Incorporating monochromation of the electron source provides sub-Angstrom spatial resolution and fully resolved C-C bonds, as shown in figure 1. [3] Correlating C-C bond lengths with DFT calculations of charge density has provided insights into bond elongation for charge depletion and bond contraction from charge accumulation.[4] This technique can also be applied to bonds at the edge of graphene to reveal triple-bond like arm-chair terminations and demonstrate that graphene edges are not always hydrogenated.[5]

Defects in graphene can be introduced with a 10nm spatial accuracy using a focused electron beam at an accelerating voltage of $80 \mathrm{kV}$ inside a TEM.[6] Vacancies are often susceptible to being filled with other atoms that diffuse across the surface of graphene. This can lead to metal dopants such as Fe filling the mono or divacancies in graphene and lead to covalently bonded substitutional and interstitial Fe dopants. [7] In certain circumstances excess carbon atoms can be incorporated into the graphene lattice via defect sites to lead to a net positive number of atoms and this leads to 'blisters' forming in graphene, shown schematically in figure 2.[8]

The defect and dopant structures evolve under the electron beam and the dynamics are compared to theoretical predictions to understand the most common forms. The results reveal the importance of C-C bond rotation in minimizing strain in graphene and the defect structures that are stable for long periods of time.

\section{References:}

[1] A.W. Robertson et al, ACS Nano 7 (2013), p. 4495.

[2] A.W. Robertson et al, Nano Letters (2014), Articles ASAP, DOI: 10.1021/n1500119p

[3] J. H. Warner et al, Science 337 (2012), p. 209.

[4] J. H. Warner et al, ACS Nano 7 (2013), p. 9860.

[5] K. He et al, Nature Communications 5 (2014), p. 3040.

[6] A. W. Robertson et al, Nature Communications 3 (2012), p. 1144. 
[7] A. W. Robertson et al, Nano Letters 13 (2013), p. 1468.

[8] A. W. Robertson et al, Nano Letters 14 (2014), p. 908.

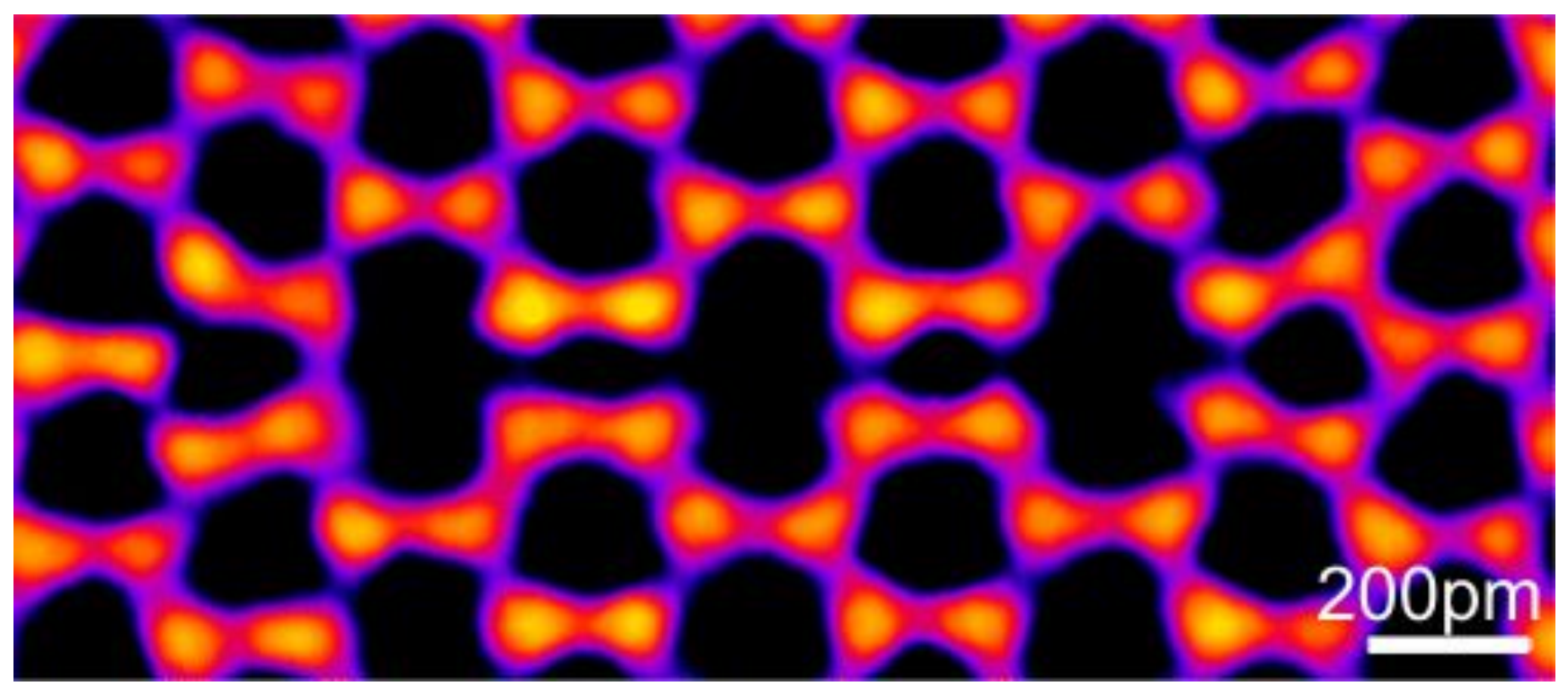

Figure 1. Monochromated aberration-corrected transmission electron microscopy image of a 6-atom vacancy defect structure in graphene with individual $\mathrm{C}$ atoms fully resolved.

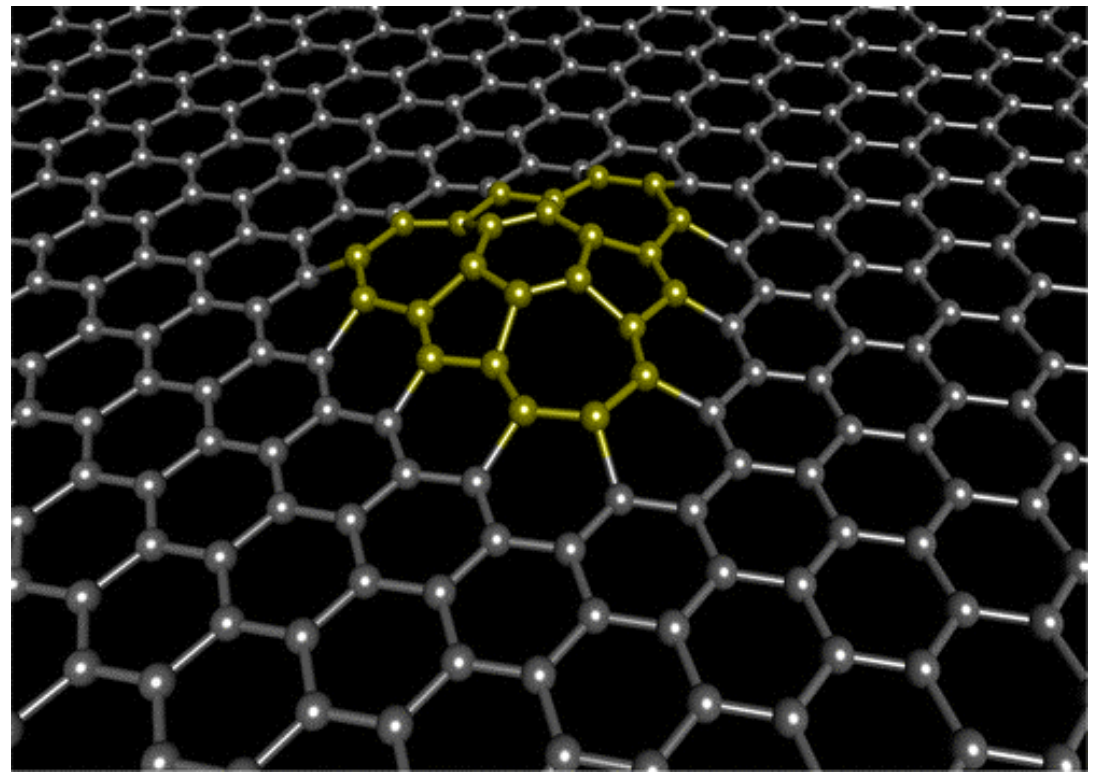

Figure 2. Schematic atomic model of 'blisters' formed in graphene from excess atoms 